\title{
Variants of Gödel's Ontological Proof in a Natural Deduction Calculus
}

\section{Kanckos, Annika}

2017

Kanckos , A \& Woltzenlogel Paleo , B 2017 , ' Variants of Gödel's Ontological Proof in a

Natural Deduction Calculus ' , Studia Logica , vol. 105 , no. 3 , pp. 553-586 . https://doi.org/10.1007/s11225-016-970

http://hdl.handle.net/10138/235481

https://doi.org/10.1007/s11225-016-9700-1

cc_by_sa

acceptedVersion

Downloaded from Helda, University of Helsinki institutional repository.

This is an electronic reprint of the original article.

This reprint may differ from the original in pagination and typographic detail.

Please cite the original version. 
Noname manuscript No.

(will be inserted by the editor)

\title{
Variants of Gödel's Ontological Proof in a Natural Deduction Calculus
}

\author{
Annika Kanckos • Bruno Woltzenlogel Paleo
}

the date of receipt and acceptance should be inserted later

\begin{abstract}
This paper presents detailed formalizations of ontological arguments in a simple modal natural deduction calculus. The first formal proof closely follows the hints in Scott's manuscript about Gödel's argument and fills in the gaps, thus verifying its correctness. The second formal proof improves the first one, by relying on the weaker modal logic KB instead of $\mathbf{S 5}$ and by avoiding the equality relation. The second proof is also technically shorter than the first one, because it eliminates unnecessary detours and uses Axiom 1 for the positivity of properties only once. The third and fourth proofs formalize, respectively, Anderson's and Bjørdal's variants of the ontological argument, which are known to be immune to modal collapse.
\end{abstract}

Keywords: Ontological Argument, Higher-Order Logics, Modal Logics, Natural Deduction.

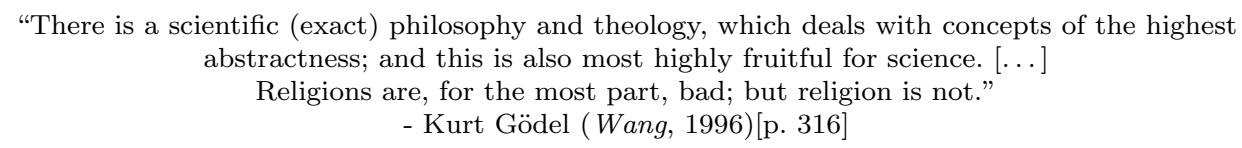

\section{Introduction}

Ontological arguments for the existence of God can be traced back at least to St. Anselm (1033-1109). His argument considers a greatest conceivable being, who must exist, because if it did not have the property of existence, then we could conceive of a greater being that,

Author order is alphabetical by surname.

Annika Kanckos

Department of Philosophy

University of Helsinki

P.O. Box 24 (Unioninkatu 40 A)

Finland

E-mail: annika.siders@helsinki.fi

+358294129241[2em]

Bruno Woltzenlogel Paleo

Theory and Logic Group

Vienna University of Technology

Austria

E-mail: bruno@logic.at

Bruno Woltzenlogel Paleo

Logic and Computation Group

College of Engineering and Computer Science

Australian National University

Australia E-mail: bruno.woltzenlogel.paleo@anu.edu.au 
in addition to other properties, also had the property of existence. St. Anselm's argument was criticized and further developed by Descartes, Leibniz and Kant, among many others.

Leibniz identified the possible existence of God as a critical missing step in St. Anselm's argument. To fill this gap, he argued that the properties of God, the perfections, are compatible. This means that it is possible to satisfy all perfections at once, which implies that the existence of a greatest conceivable being with all these properties is possible.

Gödel built on Leibniz's work (Adams, 1995) and brought the ontological argument to a modern form using a modal logic with higher-order quantification over properties. In this setting, he gave precise axioms describing the notion of positive property and defined God as a being that has all positive properties. Gödel's work was saved in his own notes (Gödel, 1970) as well as in notes by Scott (Scott, 2001), to whom he confided his proof.

The increase in formality of the ontological argument has required a development of its basic notions. Gödel's notion of positive property and Leibniz's notion of perfection differ. A formal distinction is that Leibniz's perfections are atomic whereas Gödel's positive properties can consist of combinations of atomic properties (Fitting, 2002)[p.139]. In particular, one of Gödel's axioms states that any conjunction of positive properties is itself positive. From this axiom, it is immediately deduced that the property of being God-like is positive. Intuitively, a (possibly infinite) conjunction of positive properties is deduced from the universal definition of God-likeness. This deductive inference is not formalizable in a finite first-order calculus. The interplay between universal quantification (in the definition of a God-like being) and infinite conjunctions (in Gödel's axiom for positive properties) could explain why, starting with Scott $(S c o t t, 2001)$, this axiom of Gödel has been replaced by another that simply states the positivity of the property of being god-like.

The aim of this paper is to present detailed formalizations of variants of ontological arguments in a natural deduction calculus. For a comprehensive introduction to natural deduction, the reader can consult (Prawitz, 2006) or (Gabbay, 1996). The natural deduction calculus proposed and used in this paper is an extension of a standard natural deduction calculus for (non-modal) higher-order logic (Benzmüller \& Brown \& Kohlhase, 2004) with new introduction and elimination rules for modal operators (as presented in Section 2). This calculus is sound and complete relative to the non-modal calculus extended with Axiom $\mathrm{K}$ and the Necessitation rule (as demonstrated in Subsection 2.1). There are many higher-order logics (Benzmüller $\mathscr{E}$ Brown $\mathscr{E}$ Kohlhase, 2004) and higher-order modal logics (Muskens, 2006). The calculus proposed here can be regarded as a minimalistic calculus for a rigid higher-order modal logic $\mathbf{K}$ without extensionality principles. By adding axioms for stronger modal logics (e.g. KB and S5), this calculus is sufficient to achieve this paper's aim.

The natural deduction style was chosen for four reasons. Firstly, presentations of Gödel's proof are typically either informal or formalized in other styles of proof calculi (e.g. Fitting's tableaux (Fitting, 2002) or Sobel's sentential modal calculus (Sobel, 2001)). Therefore, a formalization in natural deduction is a valuable complement to the existing presentations. Secondly, it makes the ontological proof accessible to people who are familiarized with the natural deduction style. Thirdly, as natural deduction is the style used by proof assistants such as Coq (Paulin-Mohring, 2015) and Isabelle (Nipkow \& Paulson \& Wenzel, 2002), natural deduction formalizations can be verified step-by-step in such proof assistants, and we have in fact done this (Benzmüller $\&$ Woltzenlogel Paleo, 2015a). And finally, in comparison to other kinds of proof systems (e.g. resolution, tableaux, sequent calculi), natural deduction can be considered less technical, more human-readable and more intuitive, because it relies on natural principles of reasoning such as modus ponens (implication elimination) and reasoning by assumption (implication introduction); these qualities are conducive to rendering the formalizations of ontological arguments accessible to a wider audience.

The first main contribution of this paper is a detailed formalization of Scott's version (Scott, 2001) of Gödel's ontological argument (Gödel, 1970) (as shown in Section 4) in the proposed natural deduction calculus. The second main contribution of the paper is a new proof (shown in Section 5), also in natural deduction style. In contrast to Scott's proof 
(Scott, 2001), which requires the modal logic S5, the new proof requires only the weaker modal logic KB. The new proof also does not rely on the equality relation and is much shorter. Although there have been claims (as discussed in more detail in Section 5) about the sufficiency of weaker modal logics and the superfluousness of the equality relation, formal proofs substantiating these claims were missing up to now.

A major criticism against Gödel's argument is an undesirable consequence of the stipulated axioms known as modal collapse. This is discussed in greater detail in Section 6, where a natural deduction derivation of the collapse is presented. Many recent works on the ontological argument have proposed modifications of the argument that do not entail modal collapse. In this paper we present natural deduction formalizations of two of these solutions: Anderson's emendation (in Section 7) and Bjørdal's variant (in Section 8).

\subsection{Related Work}

An early unpublished draft of Section 5 of this paper, prepared by Woltzenlogel Paleo in March 2013, served as the starting point for two distinct but complementary research endeavours. Kanckos and Woltzenlogel Paleo transformed that early draft into this paper, aiming for human-readable and yet formal natural deduction proofs. Benzmüller and Woltzenlogel Paleo, on the other hand, focused on the verification of variants of Gödel's ontological arguments using higher-order automated reasoning tools, which quickly led to many publications. The main automatically obtained results about Gödel's and Scott's proofs appeared in the European Conference on Artificial Intelligence (Benzmüller \& Woltzenlogel Paleo, 2014d) and, two years later, a detailed analysis of an inconsistency found in Gödel's axioms was published in the International Joint Conference on Artificial Intelligence (Benzmüller \& Woltzenlogel Paleo, 2016a). The automatic verification of variants by Anderson, Bjørdal and Hájek were presented in the First World Congress on Logic and Religion (Benzmüller \& Weber \& Woltzenlogel Paleo, 2015) and published in (Benzmüller \& Weber \& Woltzenlogel Paleo, 2017) and results related to the modal collapse were reported in the 4th Word Congress on the Square of Opposition (Benzmüller $\& 3$ Woltzenlogel Paleo, 2014c) and later published in (Benzmüller $\&$ Woltzenlogel Paleo, 2016b). More detailed reconstructions of Scott's variant within the interactive proof assistants Isabelle and Coq were released, respectively, in Isabelle's Archive of Formal Proofs (Benzmüller \& Woltzenlogel Paleo, 2013b) and in the Symposium of Computer Science in Russia (Benzmüller \& Woltzenlogel Paleo, 2015a). The popularity of the topic led to invited talks and corresponding invited abstracts in the proceedings of workshops (Benzmüller \& Woltzenlogel Paleo, 2014a,b, 2013a) and summer schools (Benzmüller $\&$ Woltzenlogel Paleo, 2015c,b) as well as articles targeting a more general audience (Woltzenlogel Paleo, 2013). More recently, this line of research ventured into other types of ontological arguments, and a computer-assisted reconstruction of an argument by Leibniz will appear as a chapter in a book dedicated to the 300th anniversary of Leibniz's death (Bentert \& Benzmüller \& Streit \& Woltzenlogel Paleo, 2016). The automatic verification of Gödel's and Scott's variants (Benzmüller \& Woltzenlogel Paleo, 2014d) was preceded by an automatic verification of (a non-modal version of) Anselm's ontological argument (Oppenheimer \& Zalta, 2011). However, whereas the complexity of Gödel's and Scott's arguments required the embedding of higher-order modal logics into higher-order logics and the use of higher-order automated reasoners, Anselm's argument greater simplicity enabled its verification with a first-order prover. Anselm's argument has also been formalized within the interactive proof assistant PVS (Rushby, 2013) and there has been a failed attempt to formalize Avicenna's argument in PVS as well.

\section{Natural Deduction}

The language of higher-order modal logic used here is inspired by that of Church's simple type theory (Church, 1940). 
Definition 1 Simple types are given by the following grammar:

$$
\theta, \tau \quad::=\mu|o| \theta \rightarrow \tau
$$

where $\mu$ is the atomic type for individuals, $o$ is the atomic type for propositions and $\theta \rightarrow \tau$ is the type for functions taking an argument of type $\theta$ and returning something of type $\tau$. ' $\rightarrow$ ' is assumed to be right associative.

Definition 2 Terms and formulas are given by the following grammar:

$$
\begin{aligned}
s, t::= & p_{\tau}\left|X_{\tau}\right|\left(\lambda X_{\theta} . s_{\tau}\right)_{\theta \rightarrow \tau}\left|\left(s_{\theta \rightarrow \tau} t_{\theta}\right)_{\tau}\right| \\
& \perp_{o}\left|\rightarrow_{o \rightarrow o \rightarrow o}\right| \wedge_{o \rightarrow o \rightarrow o} \mid \\
& \forall_{(\tau \rightarrow o) \rightarrow o}\left|\exists_{(\tau \rightarrow o) \rightarrow o}\right| \square_{o \rightarrow o} \mid \diamond_{o \rightarrow o}
\end{aligned}
$$

where $p_{\tau}$ and $X_{\tau}$ range over, respectively, constants and variables of type $\tau$. Parenthesis conventions, infix notation for propositional connectives and binding notation ${ }^{1}$ for quantifiers are assumed. Furthermore, subscript types are omitted when they are clear from the context. Implicit alpha-conversion is assumed: two expressions are considered to be the same modulo renaming of their bound variables. Negation $\left(\neg_{o \rightarrow 0}\right)$, disjunction $\left(\vee_{o \rightarrow o \rightarrow o}\right)$, equivalence $\left(\leftrightarrow_{o \rightarrow o \rightarrow o}\right)$, equality $\left(=_{\tau \rightarrow \tau \rightarrow o}\right)$, inequality $\left(\neq_{\tau \rightarrow \tau \rightarrow o}\right)$, are defined by $\neg A \equiv A \rightarrow \perp,(A \vee$ $B) \equiv \neg(\neg A \wedge \neg B),(A \leftrightarrow B) \equiv(A \rightarrow B) \wedge(B \rightarrow A), s=t \equiv \forall P .(P s) \leftrightarrow(P t)$ (Leibniz's equality) and $s \neq t \equiv \neg(s=t)$.

The natural deduction calculus proposed and used here has standard rules for propositional connectives and quantifiers, as shown in Figures 1 and 4. The extension to classical logic is achieved by adding a rule for double negation elimination, shown in Figure 3, and $\beta$-reduction ${ }^{2}$ is handled by the $\beta$ rule shown in Figure 5 . Finally, modal operators are handled by special new rules that insert or remove formulas from boxes, as shown in Figure 6 . A derivation is a directed acyclic graph whose nodes are formulas and whose edges correspond to applications of the inference rules. A proof of a formula $F$ is a derivation without open assumptions and having $F$ as root not inside any box.

In every inference rule, (sub)formulas in the conclusion correspond ${ }^{3}$ to formulas in the premises (even in the case of quantifier rules, where the formulas differ due to substitution). For any expression occurring both in a formula in the premise and in its corresponding (sub)formula in the conclusion, the occurrence in the premise is said to be an ancestor of the occurrence in the conclusion, and the occurrence in the conclusion is said to be a descendant of the occurrence in the premise.

Applications of the $\beta$ rule are silently omitted. Double lines are used to abbreviate propositional reasoning steps when they are tedious and too space-consuming. Dashed lines are used to refer to an axiom or theorem with the proof shown elsewhere. When proof trees are too large to fit on the page, some branches may be displayed separately. In such cases, the conclusion of the branch and the location in the main proof tree where the branch belongs are annotated with the same symbol (a subscripted $\star$ ). Dotted lines are used to indicate folding and unfolding of definitions. Furthermore, as it is inconvenient to draw boxes around large derivations in $\mathrm{IT}_{\mathrm{E}} \mathrm{X}$, formulas inside boxes are labeled with the names of the boxes surrounding them. Therefore, the boxes can be omitted without loss of information.

The calculus having only the rules shown in Figures 1, 3, 4 and 5 is named ND. This calculus is essentially the same as the simplest natural deduction calculus in the family of calculi discussed in (Benzmüller \&3 Brown \& Kohlhase, 2004). The main difference is that they take disjunction as a primitive connective instead of conjunction, and they use a sequent notation, where the assumptions are listed in the left side of the sequent. The calculus with the new rules for modal operators shown in Figure 6 is named $\mathbf{N D}_{\mathbf{K}}$.

\footnotetext{
$1 \forall x . F[x]$ and $\exists x . F[x]$ are assumed to be abbreviations for, respectively, $\forall(\lambda x . F[x])$ and $\exists(\lambda x . F[x])$.

2 Rules for $\eta$-expansion (Benzmüller $\&$ Brown $\&$ Kohlhase, 2004) are not included here, because they are not needed in the ontological arguments studied here.

3 This correspondence could be defined formally, but it would be tedious to do so.
} 
Fig. 1 Propositional rules

$$
\begin{array}{ccc} 
& \bar{A} \\
\frac{\perp}{A} \perp_{E} & \vdots \\
\frac{A}{A \wedge B} \wedge_{I} & \frac{B}{A \rightarrow B} \rightarrow_{I} & \frac{A \quad A \rightarrow B}{B} \rightarrow_{E} \\
& \frac{A \wedge B}{A} \wedge_{E_{1}} & \frac{A \wedge B}{B} \wedge_{E_{2}}
\end{array}
$$

Fig. 2 Admissible propositional rules

$$
\frac{\neg A \quad A}{\perp} \neg_{E} \quad \frac{A \leftrightarrow B \quad B}{A} \leftrightarrow_{E} \quad \frac{A \leftrightarrow B \quad A}{B} \leftrightarrow_{E}
$$

Fig. 3 Double negation elimination

$$
\frac{\neg \neg A}{A} \neg \neg E
$$

Fig. 4 Quantifier rules

$$
\frac{A[\alpha]}{\forall x_{\tau} \cdot A[x]} \forall_{I} \quad \frac{\forall x_{\tau} \cdot A[x]}{A[t]} \forall_{E} \quad \frac{A[t]}{\exists x_{\tau} \cdot A[x]} \exists_{I} \quad \frac{\exists x_{\tau} \cdot A[x]}{A[\beta]} \exists_{E}
$$

eigen-variable conditions:

if $\rho$ is a $\forall_{I}$ inference eliminating a variable $\alpha$, then any occurrence of $\alpha$ in the proof should be an ancestor of the occurrence of $\alpha$ eliminated by $\rho$ and not a descendant of a variable introduced by a $\exists_{E}$ inference;

if $\rho$ is a $\exists_{E}$ inference introducing a variable $\beta$, then any occurrence of $\beta$ in the proof should be a descendant of the occurrence of $\beta$ introduced by $\rho$ and not an ancestor of a variable eliminated by a $\forall_{I}$ inference.

Fig. 5 Reduction rules

$$
\frac{A}{B} \beta
$$

only if $B$ and $A$ are $\beta$-equivalent

(i.e. there is a $C$ such that both $B$ and $A \beta$-reduce to $C$ )

\subsection{Suitability for Rigid Higher-Order Modal Logic K}

ND is a calculus for a (non-modal) higher-order logic without functional and boolean extensionality principles (Benzmüller \& Brown \& Kohlhase, 2004). Adding the modal rules results in a calculus that is suitable for a basic rigid modal logic $\mathbf{K}$, in the sense that $\mathbf{N D}_{\mathbf{K}}$ is sound and complete relative to ND extended with Axiom $\mathrm{K}(\square(A \rightarrow B) \rightarrow(\square A \rightarrow \square B))$ and the necessitation rule (which establishes that $\square A$ is a theorem if $A$ is a theorem).

Theorem 1 ND $\mathbf{K}$ is complete relative to $\mathbf{N D}$ extended with Axiom $K$ and necessitation. 
Fig. 6 Rules for modal operators

$$
\begin{aligned}
& \frac{\omega: \begin{array}{c|}
\hline \vdots \\
A
\end{array}}{\square A} \square_{I} \\
& \frac{\square A}{{ }^{A}} \square_{E} \\
& \frac{w: \begin{array}{c}
\vdots \\
\vdots \\
A
\end{array}}{\diamond A} \diamond_{I} \\
& \frac{\diamond A}{\omega: \begin{array}{c}
A \\
\vdots
\end{array}} \diamond_{E}
\end{aligned}
$$

eigen-box condition:

a modal inference is said to access

the box immediately above or below it.

$\square_{I}$ and $\diamond_{E}$ are strong modal rules:

$\omega$ must be a fresh name for the box they access

(in analogy to the eigen-variable condition for strong quantifier rules).

Every box must be accessed by exactly one strong modal inference.

boxed assumption condition:

assumptions should be discharged within the box where they are created.

Proof The necessitation rule can be immediately simulated with the $\square_{I}$ rule. Axiom K can be derived in $\mathbf{N D}_{\mathbf{K}}$ as shown below:

$$
\frac{\frac{\overline{\square(A \rightarrow B)}}{\omega: A \rightarrow B} \square_{E} \quad \frac{\overline{\square A}}{\omega: A}{ }_{E}}{\square_{E}}
$$

Theorem $2 \mathbf{N D}_{\mathbf{K}}$ is sound relative to $\mathbf{N D}$ extended with Axiom $K$ and necessitation.

Proof It is necessary to show that $\mathbf{N D}_{\mathbf{K}}$ proofs of the following form can be translated to proofs in ND extended with the Axiom K and the necessitation rule.

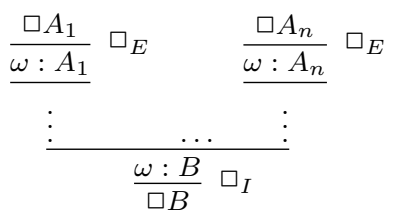

A translation to ND extended with Axiom $\mathrm{K}$ and necessitation is shown below for the case when $n=1$ :

$$
\begin{aligned}
& \overline{A_{1}} 1 \\
& \text { : } \\
& \frac{B}{A_{1} \rightarrow B} \rightarrow_{I}^{1}
\end{aligned}
$$

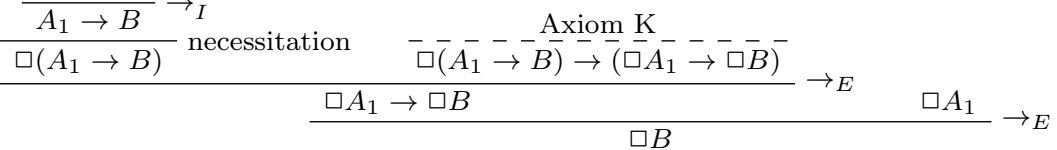

For $n>1$, the translation is a straightforward generalization: 


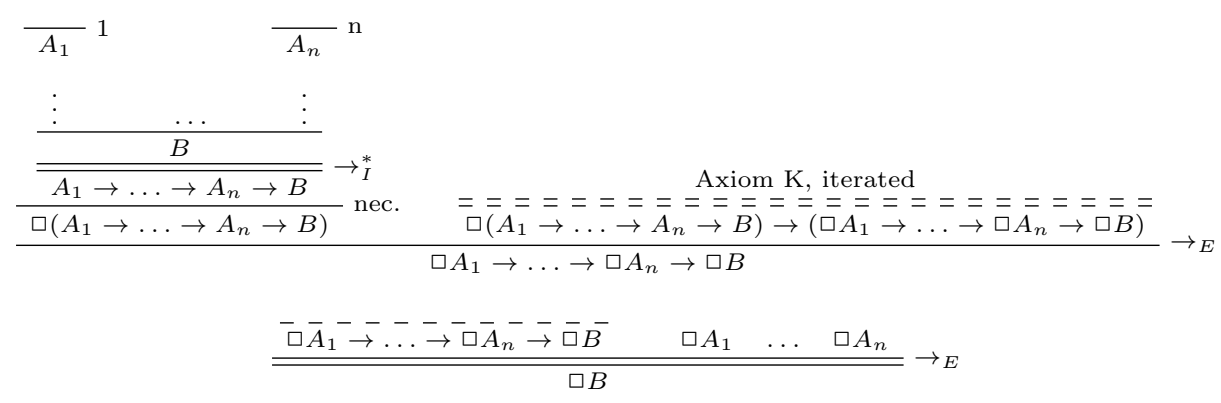

Without the restriction that every box must be accessed by exactly one strong modal inference, the calculus would be unsound for the modal logic $\mathbf{K}$. For example, the formula $\forall \phi .(\square \phi \rightarrow \diamond \phi)$ is not valid in $\mathbf{K}$ but would be provable without this restriction:

$$
\begin{gathered}
\frac{\overline{\square \phi}}{\frac{\omega: \phi}{\triangleright \phi} \square_{E}} \diamond_{I} \\
\frac{\square \phi \rightarrow \diamond \phi}{\forall \phi .(\square \phi \rightarrow \diamond \phi)} \forall_{I}^{1}
\end{gathered}
$$

This example proof is unsound according to the $\mathbf{N D}_{\mathbf{K}}$ calculus, because the eigen-box condition is violated: the box labelled by $\omega$ is not accessed by any strong inference.

The straightforward combinations of the quantifier rules of ND either with the modal rules of $\mathbf{N D}_{\mathbf{K}}$ or with Axiom $\mathrm{K}$ and the necessitation rule are suitable for a higher-order modal logic where constants and variables are rigid. From the point of view of a possible world semantics, rigidity means that their interpretation is independent of the world in which they are being interpreted. Rigidity is tacitly assumed by most works investigating the ontological argument, and is explicitly assumed here. Nevertheless, it should be noted that its adequacy for the ontological argument has already been contested (Fitting, 2002). Another assumption made here is that the quantification domains are constant (i.e. independent of the possible worlds). Neither Gödel's manuscript nor Scott's manuscript reveal whether they use constant or varying domains, and this is also the case for many variants (e.g. Hájek 1996). Nevertheless, some authors of variants of Gödel's ontological argument do explicitly state a preference for varying domains (Anderson, 1990, footnotes 11 and 14). Our choice of constant domains is motivated by simplicity. As is well-known, varying domain quantifiers (also known as actualistic quantifiers) can be simulated by constant domain quantifiers (also known as possibilistic quantifiers) guarded by a primitive existence predicate ${ }^{4}$; therefore, the formalization of ontological arguments with varying domain quantification would be feasible in the natural deduction calculus presented here as well.

\section{Some Useful Derivable Principles}

From an axiomatic point of view, modal logics stronger than $\mathbf{K}$ differ from each other with respect to which axiom schemas they admit in addition to $\mathrm{K}$ (and necessitation). Some common axiom schemas of relevance to the ontological argument are $\mathrm{T}(\square A \rightarrow A), \mathrm{B}$ $(A \rightarrow \square \diamond A), 4(\square A \rightarrow \square \square A)$ and $5(\diamond A \rightarrow \square \diamond A)$. From a semantic point of view, these axioms correspond to geometric frame conditions that must be satisfied by the accessibility relation in Kripke models (see e.g. Fitting \& Mendelsohn 1998 or Blackburn \& al. 2001, Section 3.1 on Frame definability): T corresponds to reflexivity, B corresponds to symmetry, 4 corresponds to transitivity and 5 corresponds to euclidianity. In the models of logic S5, all these axioms are satisfied; in the models of logic $\mathbf{K B}, \mathrm{B}$ is satisfied; and in the models of logic B, T and B are satisfied.

\footnotetext{
${ }^{4}$ Formally, $\forall^{e} x . F[x] \equiv \forall x . E(x) \rightarrow F[x]$ and $\exists^{e} x . F[x] \equiv \exists x . E(x) \wedge F[x]$.
} 
In this section, natural deduction proofs of three convenient derived modal principles are presented. These principles are used in the ontological proofs formalized in the next sections. Although they are well-known among modal logicians, we include their proofs here in order to have a self-contained and fully formal presentation of the ontological proofs.

The distribution principle can be seen as a form of modus ponens within the scope of modalities: if $A \rightarrow B$ holds in all accessible worlds and $A$ holds in an accessible world, then $B$ holds in an accessible world. This principle is provable in the modal logic $\mathbf{K}$.

\section{Lemma (Distribution Principle)}

$$
\square(A \rightarrow B) \rightarrow(\diamond A \rightarrow \diamond B)
$$

Proof

$$
\begin{aligned}
& \frac{\frac{\overline{\square(A \rightarrow B)}}{\omega: A \rightarrow B} \square_{E} \quad \frac{\overline{\diamond A}}{\omega: A} \diamond_{E}}{\frac{\omega: B}{\diamond B} \diamond_{I}} \rightarrow_{E} \\
& \frac{\frac{\diamond B}{\triangleright A \rightarrow \diamond B} \rightarrow_{I}^{1}}{\square(A \rightarrow B) \rightarrow(\diamond A \rightarrow \diamond B)} \rightarrow{ }_{I}^{2}
\end{aligned}
$$

Brouwer's reduction principle is derivable in modal logic $\mathbf{K B}$, using Axiom B. The proofs of Gödel and Scott (Section 4) do not make direct use of this principle, but the new proof presented in Section 5 does.

\section{Lemma (Brouwer's Reduction Principle)}

$$
\diamond \square A \rightarrow A
$$

Proof

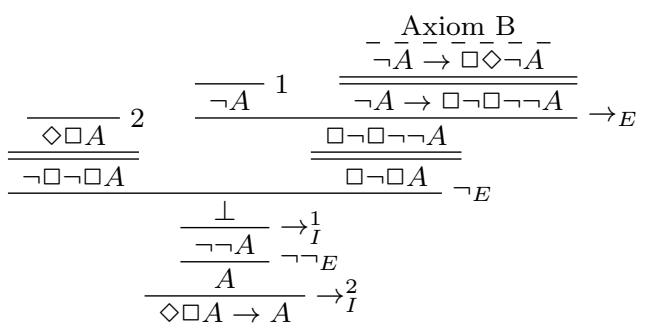

In the modal logic S5, a sequential iteration of modalities can be collapsed to the last modality in the sequence. In other words, the following principles hold in S5:

$$
\begin{aligned}
& -\diamond^{n} \square A \rightarrow \square A \\
& -\square^{n} \diamond A \rightarrow \diamond A \\
& -\diamond^{n} \diamond A \rightarrow \diamond A \\
& -\square^{n} \square A \rightarrow \square A
\end{aligned}
$$

Below we exhibit a natural deduction proof for the first principle listed above when $n=1$. Informally, this particular case can be read as the claim that anything that is possibly necessary is in fact necessary, and it is sufficient for the ontological proofs of Gödel and Scott. The proof of this principle depends on the previously proven Brouwer's reduction principle, on the Modal Axiom 5, and on K, which was shown to be derivable in the proof of Theorem 1.

\section{Lemma (Iteration Principle)}

$$
\diamond \square A \rightarrow \square A
$$




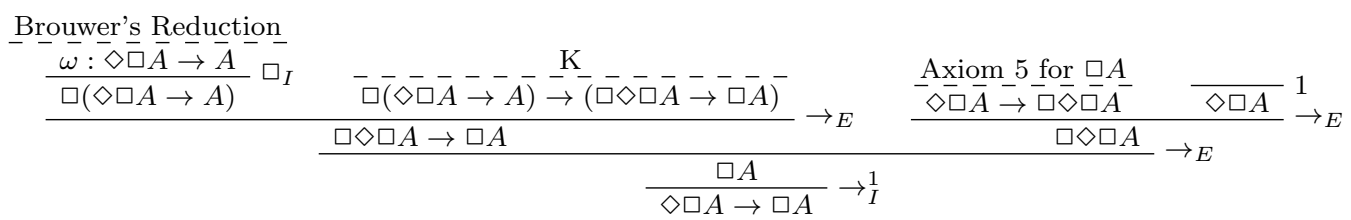

The reflexivity of equality is a theorem in higher-order logics where equality is defined as Leibniz's equality. A natural deduction proof can be easily constructed, as shown below.

\section{Lemma (Reflexivity)}

$$
\forall x \cdot x=x
$$

Proof

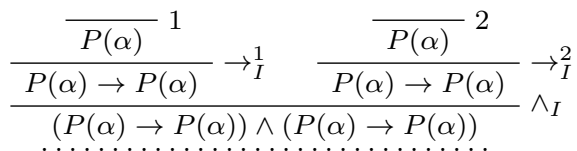

$$
\begin{aligned}
& \frac{P(\alpha) \leftrightarrow P(\alpha)}{\forall P . P(\alpha) \leftrightarrow P(\alpha)} \forall_{I} \\
& \frac{\alpha=\alpha}{\forall x . x=x} \forall I
\end{aligned}
$$

\section{Scott's Proof in Natural Deduction}

In this section we present a detailed formalization of Scott's proof in the natural deduction calculus defined in Section 2. The reasoning in Scott's manuscript has been reproduced step-by-step and reasoning gaps are completed by using the deduction steps considered most natural. All derived formulas that do appear in Scott's manuscript are marked with $\mathrm{a} \dagger$ here. Unmarked formulas were derived in the process of filling the gaps between the marked formulas.

Scott's version of Gödel's proof depends on five axioms that circumscribe the notion of positive property, with positivity being denoted by the undefined second-order predicate symbol $P$. Additionally, three definitions are used for establishing the notions of God-like, essence and necessary existence. Technically, these definitions only abbreviate certain complex formulas. The argument would still go through if all defined symbols were replaced by the complex formulas they define. This observation is particularly relevant in the case of necessary existence. Since this notion of "existence" is just an abbreviation, Gödel's argument is not susceptible to Kant's criticism against Anselm's argument (that existence should not be treated as a predicate). In Gödel's proof, existence is properly denoted by the existential quantifier. It is, therefore, unfortunately misleading to refer to the defined predicate symbol $E$ as "necessary existence", when in fact it is just a convenient abbreviation.

In addition to the five axioms and three definitions that pertain specifically to the argument, the proof also uses S5's Iteration principle (in the proof of Lemma 2) and the lemma of reflexivity for equality (in the proof of Theorem 1). The use of reflexivity could be avoided, but we chose to use it because it allows the construction of a shorter proof.

Axiom 1 Either a property or its negation ${ }^{5}$ is positive, but not both:

$$
\forall \varphi \cdot(P(\neg \varphi) \leftrightarrow \neg P(\varphi))
$$

Axiom 2 A property necessarily implied by a positive property is positive:

$$
\forall \varphi \cdot \forall \psi \cdot((P(\varphi) \wedge \square \forall x \cdot(\varphi(x) \rightarrow \psi(x))) \rightarrow P(\psi))
$$

\footnotetext{
5 Throughout the paper, $\neg \varphi$ is an abbreviation for $\lambda x . \neg \varphi(x)$
} 
Theorem 1 Positive properties are possibly exemplified:

$$
\forall \varphi \cdot(P(\varphi) \rightarrow \diamond \exists x \cdot \varphi(x))
$$

Proof

$$
\begin{aligned}
& \text { Reflexivity }
\end{aligned}
$$

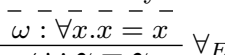

$$
\begin{aligned}
& \frac{\omega: \gamma=\gamma}{\omega: \rho(\gamma) \rightarrow \gamma=\gamma} \rightarrow_{I}
\end{aligned}
$$

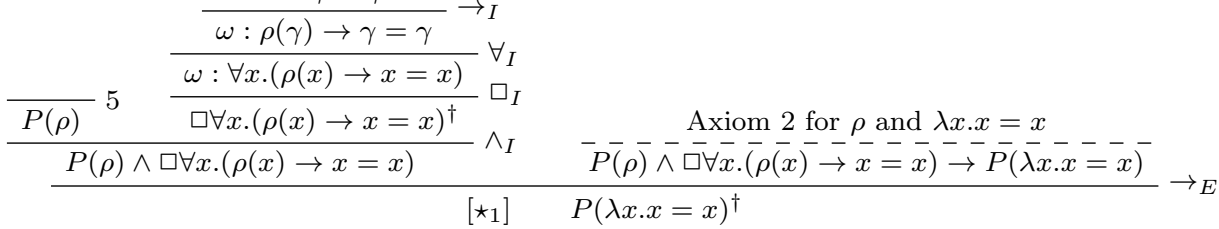

$$
\begin{aligned}
& \frac{\frac{\square \forall x \cdot \neg \rho(x)}{\frac{\square: \rho(\beta)}{\omega: \forall x . \neg \rho(x)}} \square_{E}}{\frac{\omega: \perp}{\omega: \neg \rho(\beta)}} \forall_{E}
\end{aligned}
$$

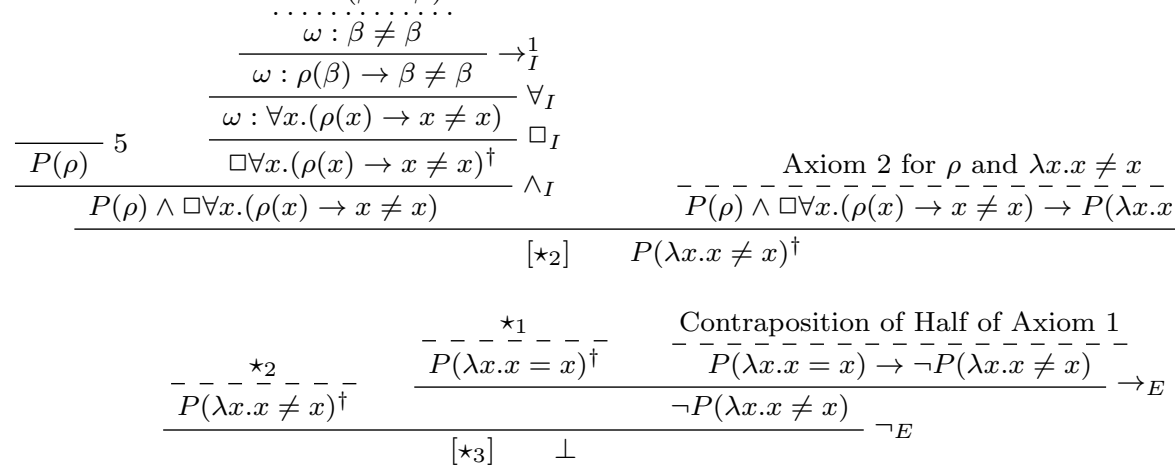

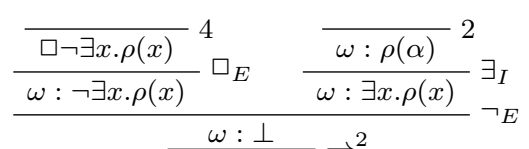

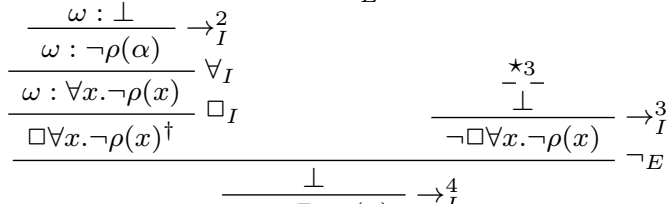

$$
\begin{aligned}
& \begin{array}{c}
\frac{\perp}{\frac{\neg \square \neg \exists x . \rho(x)}{\diamond \exists x . \rho(x)}} \rightarrow_{I}^{4} \\
\frac{P(\rho) \rightarrow \diamond \exists x \cdot \rho(x)}{\forall \varphi .(P(\varphi) \rightarrow \diamond \exists x \cdot \varphi(x))} \rightarrow_{I}^{5}
\end{array}
\end{aligned}
$$

Definition 1 A God-like being possesses all positive properties:

$$
G(x) \equiv \forall \varphi \cdot(P(\varphi) \rightarrow \varphi(x))
$$

Axiom 3 The property of being god-like is positive:

$$
P(G)
$$

Corollary 1 Possibly, God exists:

$$
\diamond \exists x \cdot G(x)
$$


Proof

$$
\begin{aligned}
& \text { Axiom } 3 \quad \text { Theorem } 1 \text { for } \mathrm{G} \\
& \frac{\bar{P}(G) \quad P(\bar{G}) \rightarrow \diamond \exists x \cdot \bar{G}(x)}{\diamond \exists x \cdot G(x)} \rightarrow E
\end{aligned}
$$

Axiom 4 Positive properties are necessarily positive:

$$
\forall \varphi .(P(\varphi) \rightarrow \square P(\varphi))
$$

Definition 2 An essence of an individual is a property possessed by it and necessarily implying any of its properties:

$$
\varphi \text { ess } x \equiv \varphi(x) \wedge \forall \psi \cdot(\psi(x) \rightarrow \square \forall x .(\varphi(x) \rightarrow \psi(x)))
$$

Theorem 2 Being god-like is an essence of any God-like being:

$$
\forall x . G(x) \rightarrow G \text { ess } x
$$

Proof

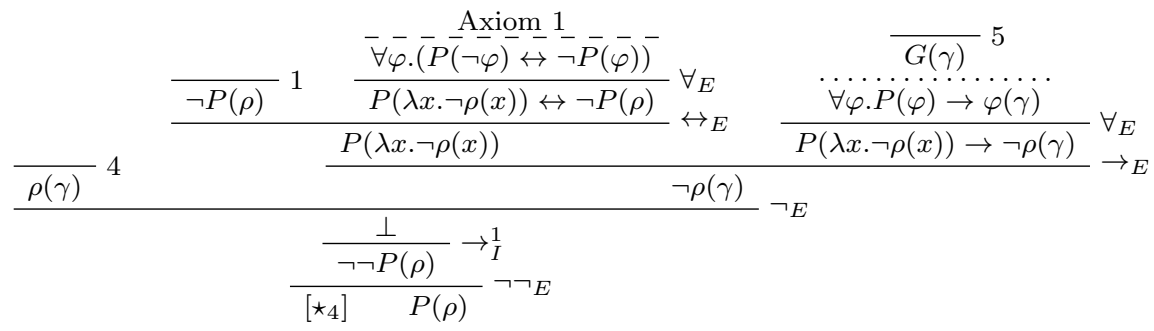

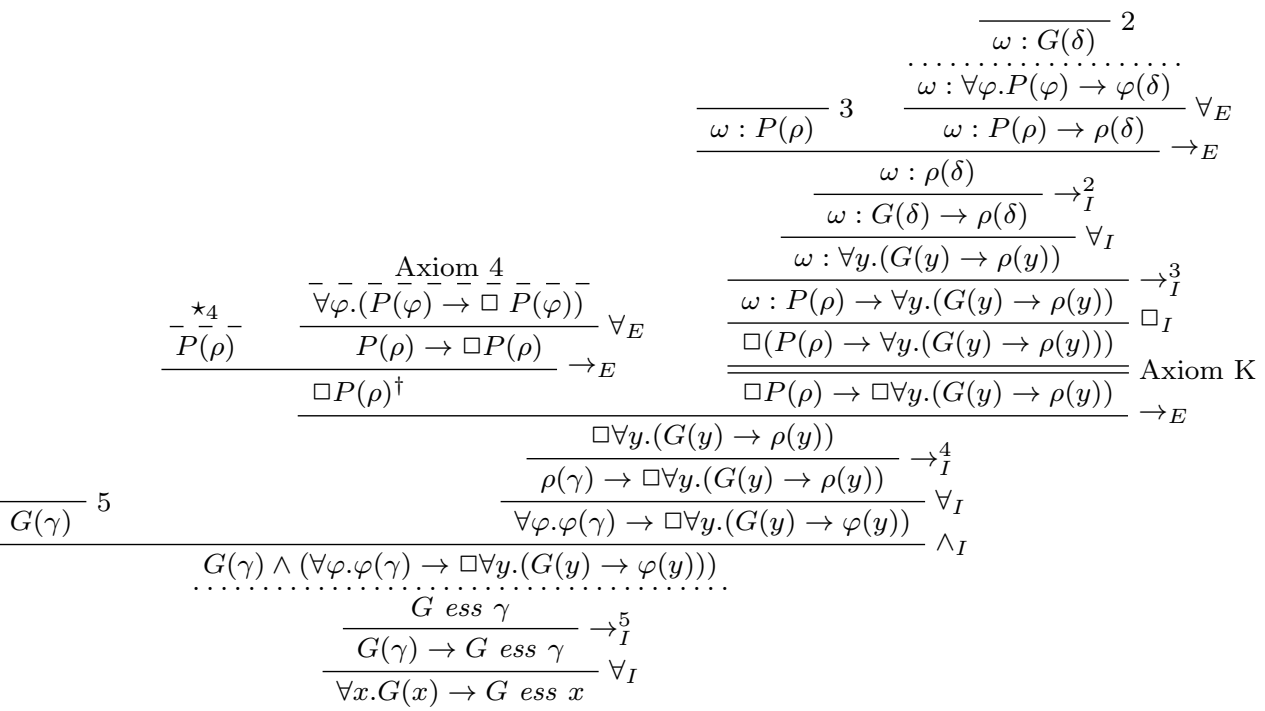

Definition 3 Necessary existence of an individual is the necessary exemplification of all its essences:

$$
E(x) \equiv \forall \varphi \cdot(\varphi \text { ess } x \rightarrow \square \exists y \cdot \varphi(y))
$$

Axiom 5 Necessary existence is a positive property:

$$
P(E)
$$

Lemma 1 If there is a God-like being, then there is a God-like being necessarily:

$$
\exists z \cdot G(z) \rightarrow \square \exists x \cdot G(x)
$$




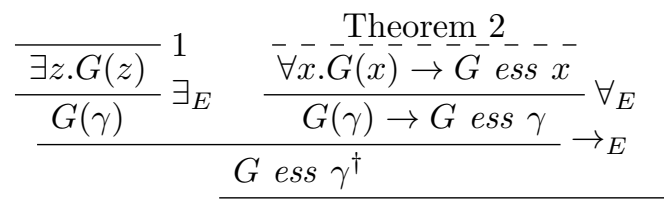

$$
\begin{aligned}
& \underline{\operatorname{Axiom}}_{\underline{P}(E)} \frac{\ddot{\forall} \boldsymbol{5} . P(\varphi) \rightarrow \varphi(\gamma)}{P(E) \rightarrow E(\gamma)} \rightarrow_{E} \\
& E(\gamma)^{\dagger} \ldots \ldots \ldots \\
& \frac{\dddot{\forall} \varphi . \varphi \text { ess } \gamma \rightarrow \square \exists x . \varphi(x)}{G \text { ess } \gamma \rightarrow \square \exists x . G(x)} \forall_{E} \\
& \frac{\square \exists x . G(x)}{\exists z . G(z) \rightarrow \square \exists x . G(x)} \rightarrow{ }_{I}^{1}
\end{aligned}
$$

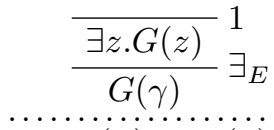

Lemma 2 If the existence of a God-like being is possible, then it is necessary:

$$
\diamond \exists z . G(z) \rightarrow \square \exists x . G(x)
$$

Proof

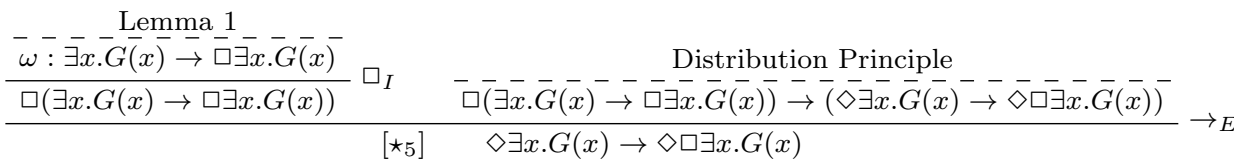

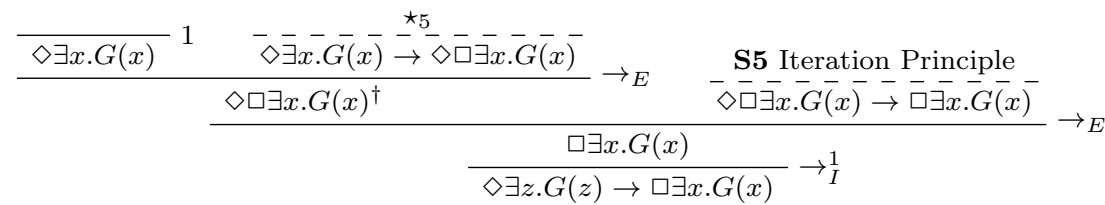

Theorem 3 Necessarily, there exists a God-like being:

$$
\square \exists x . G(x)
$$

Proof

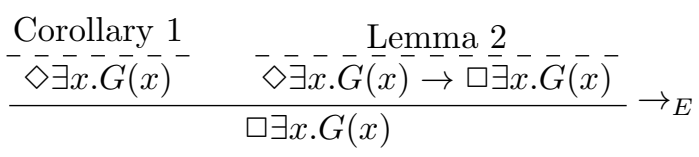

\section{A New Proof}

In this section we present a new proof in KB. This proof uses exactly the same axioms and definitions of Scott's proof shown in the previous sections, but it uses neither the Axiom of reflexivity for equality nor S5's Modal iteration principle. Instead, it relies only on Brouwer's reduction principle (for Theorem 3). The new proof is also shorter than Scott's proof.

Axiom 1 Either a property or its negation is positive, but not both:

$$
\forall \varphi .(P(\neg \varphi) \leftrightarrow \neg P(\varphi))
$$

Axiom 2 A property necessarily implied by a positive property is positive:

$$
\forall \varphi . \forall \psi \cdot((P(\varphi) \wedge \square \forall x .(\varphi(x) \rightarrow \psi(x))) \rightarrow P(\psi))
$$


Theorem 1 Positive properties are possibly exemplified:

$$
\forall \varphi \cdot(P(\varphi) \rightarrow \diamond \exists x \cdot \varphi(x))
$$

Proof

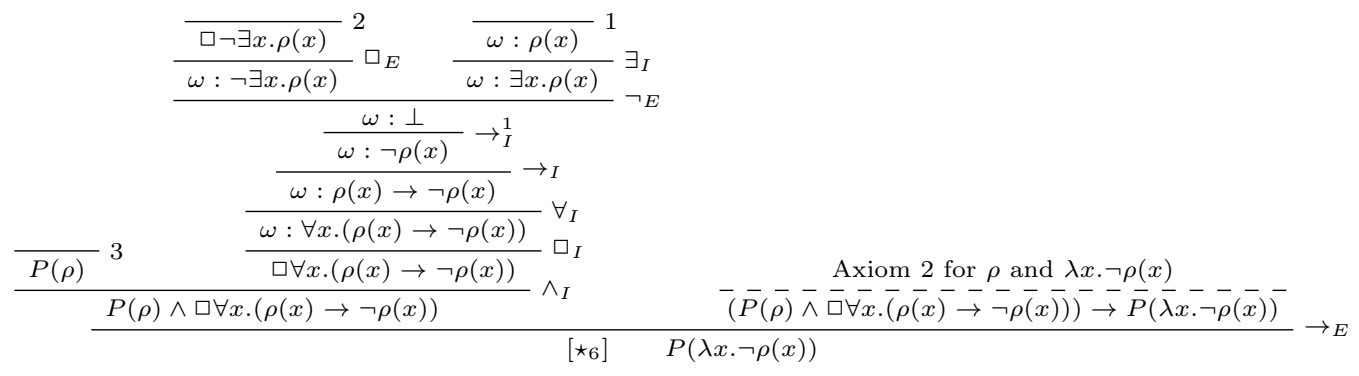

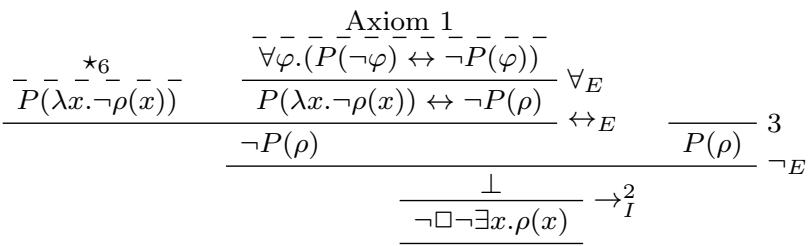

$$
\begin{aligned}
& \frac{\frac{\overline{\diamond \exists x \cdot \rho(x)}}{P(\rho) \rightarrow \diamond \exists x \cdot \rho(x)} \rightarrow_{I}^{3}}{\forall \varphi .(P(\varphi) \rightarrow \diamond \exists x \cdot \varphi(x))} \forall_{I}
\end{aligned}
$$

Comparing this new proof of Theorem 1 with the proof of Theorem 1 indicated by Gödel and Scott, it is noticeable that the new proof is significantly shorter. The key idea to obtain a shorter proof is to instantiate the second universally quantified property of Axiom 2 by $\lambda x . \neg \rho(x)$ instead of using the relation symbols $=$ or $\neq$ as Gödel and Scott did. This eliminates the reliance of the ontological argument on the presence of $=$ and $\neq$ in the logical language. Furthermore, Axiom 2 is then needed only once.

Although the simpler proof of Theorem 1 presented here was developed independently, it seems conceptually related to a possible simplification briefly described by Anderson (Anderson, 1990, footnote 2) and attributed by him to an anonymous referee. The simpler proof of Theorem 1 is not particularly special. In fact, it is probably the most likely proof that any well-trained logician would construct, if asked to derive Theorem 1 from Axioms 1 and 2 using methodical proof search techniques. Modern techniques usually rely on unification to compute or guess good instances for weakly quantified variables, and therefore they would never result in instantiations containing $=$ and $\neq$, which are symbols that do not appear anywhere in the axioms or in the theorems to be proved. We may wonder why Gödel preferred to use those unusual instantiations. It might be an interesting question from a historical point of view, suggesting that there may have been an evolution in the way logicians construct formal proofs, or from a philosophical point of view, if Gödel's preference was philosophically motivated.

Definition 1 A God-like being possesses all positive properties:

$$
G(x) \equiv \forall \varphi \cdot(P(\varphi) \rightarrow \varphi(x))
$$

Axiom 3 The property of being god-like is positive:

$$
P(G)
$$

Corollary 1 Possibly, a God-like being exists:

$$
\diamond \exists x . G(x)
$$


Proof The proof of this corollary is identical to the proof shown in Section 4.

Axiom 4 Positive properties are necessarily positive:

$$
\forall \varphi .(P(\varphi) \rightarrow \square P(\varphi))
$$

Definition 2 An essence of an individual is a property possessed by it and necessarily implying any of its properties:

$$
\varphi \text { ess } x \equiv \varphi(x) \wedge \forall \psi \cdot(\psi(x) \rightarrow \square \forall x .(\varphi(x) \rightarrow \psi(x)))
$$

At this point, Gödel and Scott proceed to prove Theorem 2, which is then used to prove Lemma 1. However, a close inspection of Scott's proof of Lemma 1 reveals that Theorem 2 is an unnecessary detour. Therefore, we do not include it in the new proof.

Definition 3 Necessary existence of an individual is the necessary exemplification of all its essences:

$$
E(x) \equiv \forall \varphi \cdot(\varphi \text { ess } x \rightarrow \square \exists y \cdot \varphi(y))
$$

Axiom 5 Necessary existence is a positive property:

$$
P(E)
$$

The new proof of Lemma 1 does not rely on Theorem 2. Overall, it is slightly shorter than Scott's proofs of Theorem 2 and Lemma 1 combined, but a large part of the new proof of Lemma 1 is structurally very similar to Scott's proof of Theorem 2, and their underlying intuitive ideas are still essentially the same. However, despite being an unnecessary detour from a technical point of view, Theorem 2 is very interesting from a philosophical perspective. It breaks an otherwise long proof of Lemma 1 in a point that facilitates comprehension by humans at an intuitive and informal level.

This phenomenon is intriguing: the new proof of Lemma 1 is technically simpler (because it has fewer inferences), but Scott's proof of Lemma 1 with Theorem 2 can be considered intuitively simpler (because it is easier to understand). This constitutes an interesting case for Hilbert's 24th Problem (Thiele, 2003), which asks for criteria to properly compare the simplicity of proofs. While Hilbert had mathematical proofs in mind, insights into the 24th problem could be gained by analyzing philosophical proofs as well. As Gödel suggested, "a scientific (exact) philosophy and theology [...] is also most highly fruitful for science".

Lemma 1 If there is a God-like being, then there is a God-like being necessarily:

$$
\exists z \cdot G(z) \rightarrow \square \exists x \cdot G(x)
$$

Proof

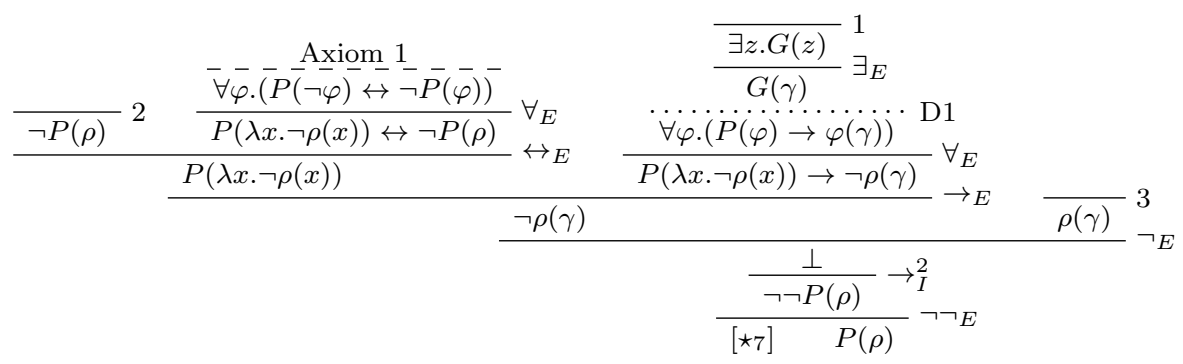




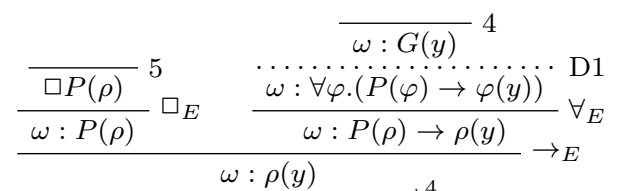

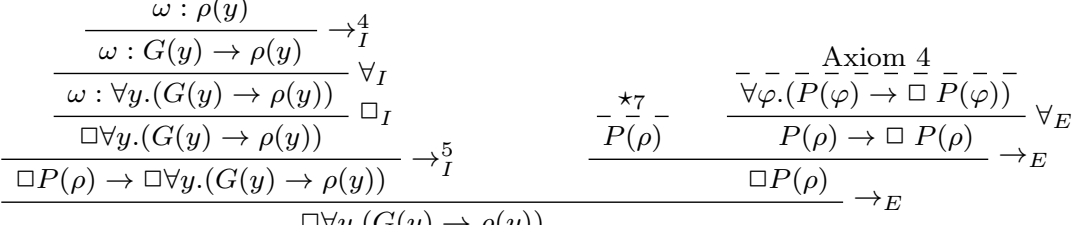

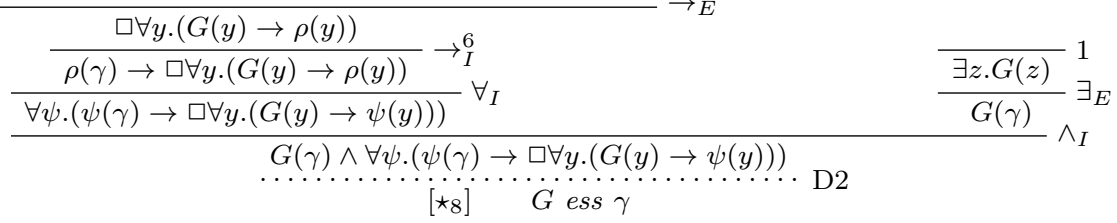

$$
\begin{aligned}
& \frac{\dddot{\exists} z \cdot G(z)}{G(\gamma)} \exists_{E}
\end{aligned}
$$

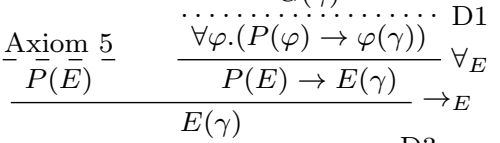

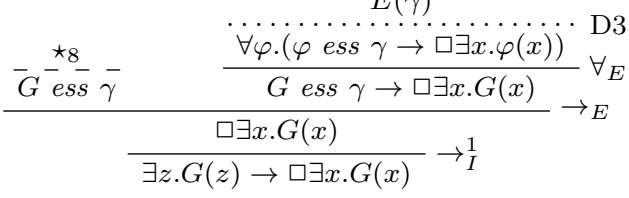

Lemma 2 also turns out to be a superfluous detour and therefore the new proof does not include it. Inspecting Scott's proof of Lemma 2, we see that an important step is the derivation of $\square \exists x . G(x)$ from $\diamond \square \exists x . G(x)$ and S5's Iteration principle. Instead, we can derive $\exists x . G(x)$ from $\diamond \square \exists x . G(x)$ and Brouwer's reduction theorem and then derive $\square \exists x . G(x)$ from $\exists x . G(x)$ by the $\square_{I}$ (necessitation) inference rule. This is precisely what the new proof of Theorem 3 does.

Theorem 3 Necessarily, there exists a God-like being:

$$
\square \exists x . G(x)
$$

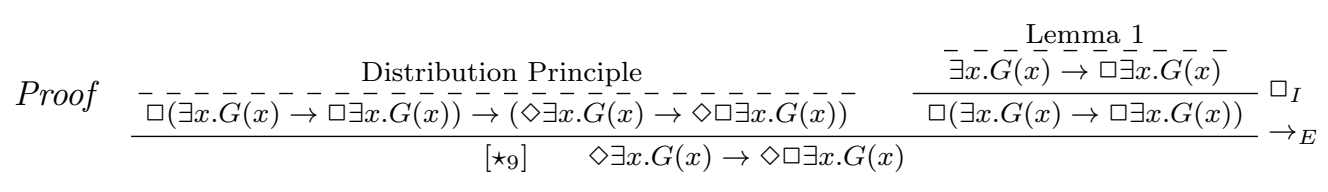

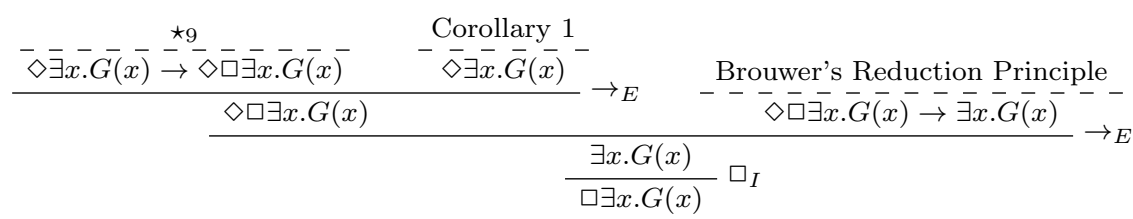

Since the Iteration principle requires the strong modal logic S5 while Brouwer's reduction principle requires only the much weaker modal logic $\mathbf{K B}$, the possibility of proving Theorem 3 using Brouwer's reduction principle instead of S5's Iteration principle is philosophically profound. There have been many discussions of what modal axioms are needed for the ontological argument. In their manuscripts, Gödel and Scott do not write explicitly which logic they use. However, a proof step in their notes clearly relies on S5's Iteration principle. Anderson (Anderson, 1990, footnote 5) cites several articles questioning the adequacy of S5 for the ontological argument and conjectures that the ontological argument could be 
restricted to the weaker modal logic B. Sobel (Sobel, 2001, p. 152) acknowledges Anderson's conjecture, claims that it is correct and tries to informally explain how his formal proofs could be modified to rely on B instead of S5. Nevertheless, neither Anderson nor Sobel presented any formal proof relying only on $\mathbf{B}$. The new formal proof presented in this section relies on $\mathbf{K B}$, which is weaker than $\mathbf{B}$.

Curiously, in the new proof of Theorem 3, the actual existence of a God-like being $(\exists x \cdot G(x))$ is proven before its necessity $(\square \exists x \cdot G(x))$. This contrasts with Gödel's proof, where the actual existence is never actually proven (although it could, nevertheless, be derived as a trivial corollary by applying the modal Axiom T).

\section{Modal Collapse}

A major criticism against Gödel's proof is that its axioms lead to the so-called modal collapse (Sobel, 1987): it is possible to prove that everything that is the case is so necessarily, and hence actuality, possibility and necessity coincide (Sobel, 2001)[Ch. 4, section 6, theorems 9 and 10]. That is: for all propositions $A$,

$$
A \leftrightarrow \diamond A \leftrightarrow \square A
$$

Below we show natural deduction derivations of the modal collapse, thereby confirming that it holds ${ }^{6}$ for the axioms used in the previous sections. We show only the strongest implication above. The others are easy corollaries in sufficiently strong modal logics (e.g. where, for instance, $\square A \rightarrow \diamond A$ or $\diamond \top$ holds).

Theorem 4 For all propositions A, the following modal collapse proposition is provable:

$$
A \rightarrow \square A
$$

$$
\begin{aligned}
& \overline{\forall y .}\left(\stackrel{\text { Theorem }}{2}{ }^{2}(y)-\bar{G} \text { ess } y\right) \\
& \frac{\not \forall y .(G(y) \rightarrow G(y) \wedge \forall \forall .(\psi(y) \rightarrow \square \forall x .(G(x) \rightarrow \psi(x))))}{\frac{\forall y .(G(y) \rightarrow \forall \psi \cdot(\psi(y) \rightarrow \square \forall x .(G(x) \rightarrow \psi(x))))}{}}
\end{aligned}
$$

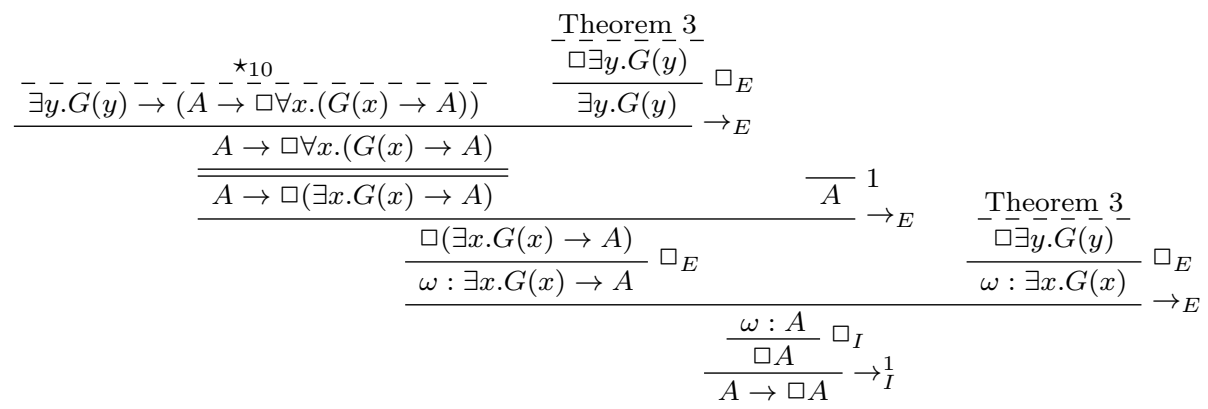

In Gödel's ontological proof, we are proving a restricted modal collapse, which applies to one specific formula, the existence of a God-like being. The interest in the proof naturally decreases if a consequence of the axiomatization is a modal collapse for all formulas. Therefore, an improvement would be obtained if the modal collapse was limited only to the property of being god-like or at least to a restricted collection of properties. Several solutions to the problem of modal collapse have been proposed. Fitting, for instance, has argued that greater

\footnotetext{
6 It is well-known and uncontroversial that the modal collapse holds. We show a natural deduction proof here merely for the sake of self-containment and so that the proof becomes more accessible to readers who might be more familiarized with natural deduction than with Sobel's proof system.
} 
care has to be taken with the semantics of higher-order modal logics. Quantified variables may be rigid or flexible; and properties may be treated as intensional or extensional. Making the right choices may prevent the modal collapse (Fitting, 2002, Sections 11.9 and 11.10). Koons (Koons, 2006), Anderson (Anderson, 1990, p. 292) and Sobel (Sobel, 2001, p. 133) also discuss the idea that the notion of property over which quantification is allowed might be too general and restrictions might be appropriate. Two alternative and more conservative solutions, requiring only modifications of the axioms and definitions but no change in the logic, are discussed in greater detail in the next sections.

\section{Anderson's Emendation in Natural Deduction}

Anderson's solution (Anderson, 1990) modifies the definitions of God-like being and essence, and eliminates half of Axiom 1 (replacing the equivalence by an implication).

Axiom 1 (Emended) If the negation of a property is positive, the property is not positive:

$$
\forall \varphi \cdot(P(\neg \varphi) \rightarrow \neg P(\varphi))
$$

Axiom 2 (As in Scott's) A property necessarily implied by a positive property is positive:

$$
\forall \varphi \cdot \forall \psi \cdot((P(\varphi) \wedge \square \forall x .(\varphi(x) \rightarrow \psi(x))) \rightarrow P(\psi))
$$

Theorem 1 Positive properties are possibly exemplified:

$$
\forall \varphi \cdot(P(\varphi) \rightarrow \diamond \exists x \cdot \varphi(x))
$$

Proof This proof is omitted, because any of the proofs of Theorem 1 in Sections 4 and 5 would suffice to prove this theorem with only a small modification: the equivalence of Axiom 1 would not need to be reduced to an implication by conjunction elimination.

Definition 1 (Emended) A God-like being necessarily possesses those and only those properties which are positive:

$$
G(x) \equiv \forall \varphi \cdot(P(\varphi) \leftrightarrow \square \varphi(x))
$$

Axiom 3 (As in Scott's) The property of being god-like is positive:

$$
P(G)
$$

Corollary 1 Possibly, a God-like being exists:

$$
\diamond \exists x \cdot G(x)
$$

Proof This proof is omitted, because it is identical to the proof of the same corollary shown in Sections 4 and 5.

Axiom 4 (As in Scott's) Positive properties are necessarily positive:

$$
\forall \varphi .(P(\varphi) \rightarrow \square P(\varphi))
$$

Definition 2 (Emended) An essence of an individual is a property necessarily implying those and only those properties necessarily possessed by it:

$$
\varphi \text { ess } x \equiv \forall \psi \cdot(\square \psi(x) \leftrightarrow \square \forall x \cdot(\varphi(x) \rightarrow \psi(x)))
$$

Definition 3 (As in Scott's) Necessary existence of an individual is the necessary exemplification of all its essences:

$$
E(x) \equiv \forall \varphi \cdot(\varphi \text { ess } x \rightarrow \square \exists y \cdot \varphi(y))
$$


Axiom 5 (As in Scott's) Necessary existence is a positive property:

$$
P(E)
$$

Because of Anderson's emendations to Axiom 1 and Definitions 1 and 2, the proof of Lemma 1 has to be modified ${ }^{7}$ as well. The proof shown below has been modified as little as possible. Interestingly, in order to eliminate the extra box modality in Anderson's emended Definition 1, the modified proof below uses the modal axiom T. Likewise, to obtain the extra box in the emended Definition 2, Axioms 2 and 3 were used.

Lemma 1 If there is a God-like being, then there is a God-like being necessarily:

$$
\exists z \cdot G(z) \rightarrow \square \exists x . G(x)
$$

Proof

$$
\begin{aligned}
& \frac{\overline{\exists z \cdot G(z)}^{1}}{{ }^{\left.\star_{11}\right]} G(\gamma)} \exists_{E}
\end{aligned}
$$

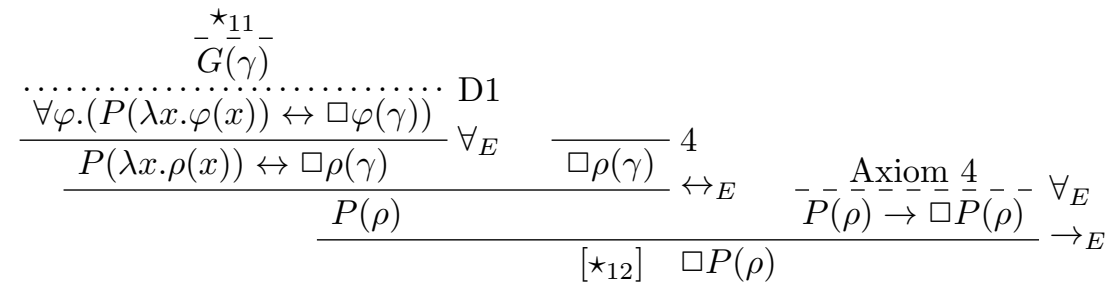

$$
\begin{aligned}
& \overline{\omega: G(y)}^{2}
\end{aligned}
$$

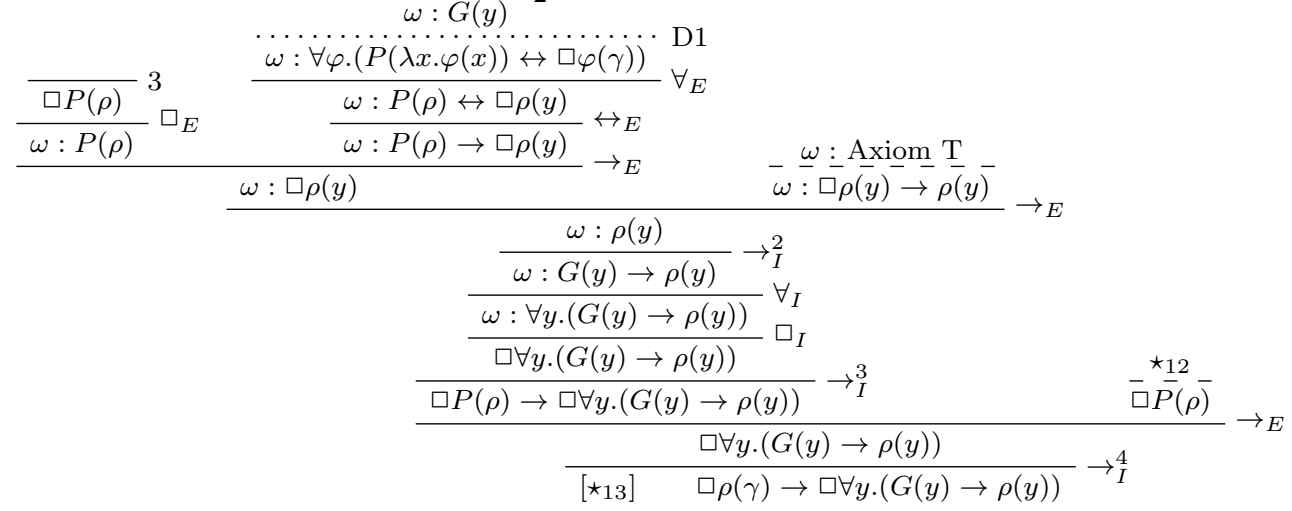

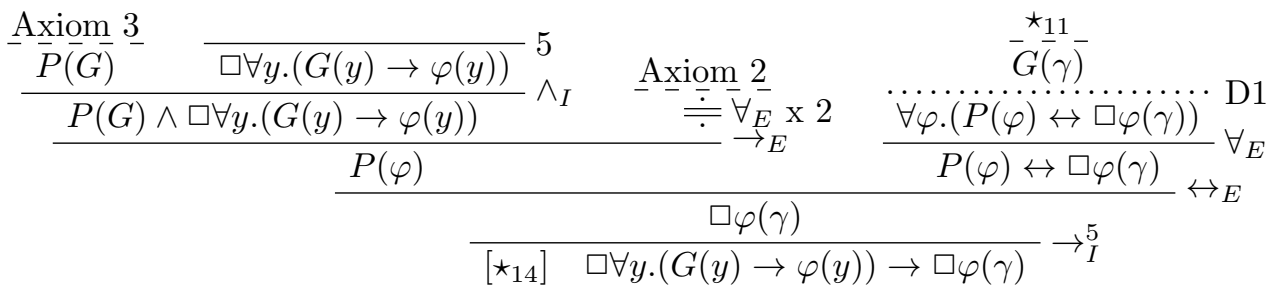

$$
\begin{aligned}
& {\left[\star_{14}\right] \quad \square \forall y \cdot(G(y) \rightarrow \varphi(y)) \rightarrow \square \varphi(\gamma) \rightarrow I}
\end{aligned}
$$

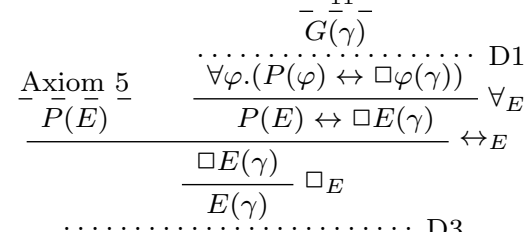

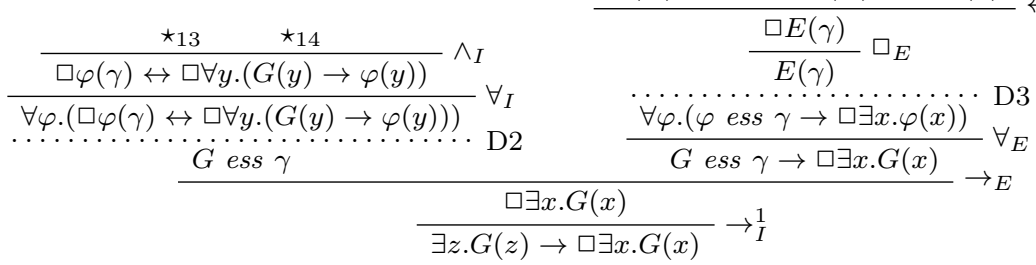

\footnotetext{
7 Due to lack of space, the instantiation of Axiom 2 is omitted in the emended proof of Lemma 1.
} 
Theorem 3 Necessarily, there exists a God-like being:

$$
\square \exists x . G(x)
$$

Proof This proof is identical to the proof of Theorem 3 shown in Section 5.

Hájek (Hájek, 1996) claimed that Anderson's emendation makes Axioms 4 and 5 derivable from the other axioms and definitions. Discussions about the redundancy of these two axioms started with an earlier (and wrong) claim by Magari (Magari, 1988) w.r.t. Scott's variant, and the redundancy was the topic of a long controversy between Hájek and Anderson (Benzmüller 83 Weber $\&$ Woltzenlogel Paleo, 2017). As confirmed in Subsection 7.1, Hájek's claim is correct. Nevertheless, the proof presented above still uses Axioms 4 and 5, since this section's intention is to show that Anderson's emendation requires only a minimal modification of the proofs presented in the previous sections.

\subsection{A Shorter Proof without Superfluous Axioms}

In order to construct a natural deduction proof of theorem 3 using only Anderson's Axioms 1, 2 and 3 as well as Anderson's Definition 1, it suffices to modify the proof of Lemma 1, which is the only part of the argument that depended on Axioms 4 and 5 and Definitions 2 and 3. The modified proof is shown below. In contrast to the previous proof, the shorter proof does not use the modal axiom T. Therefore, modal logic KB is sufficient for Anderson's emendation as well.

Lemma 1 If there is a God-like being, then there is a God-like being necessarily:

$$
\exists z . G(z) \rightarrow \square \exists x . G(x)
$$

Proof

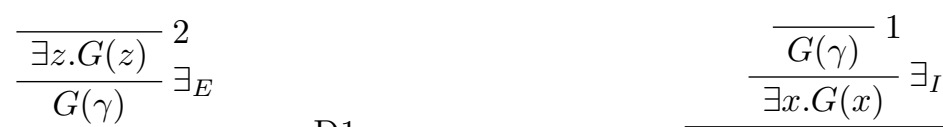

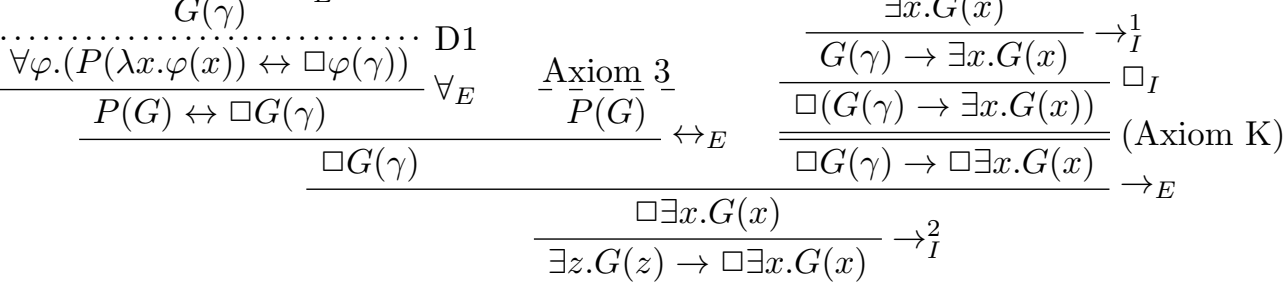

Proofs of other lemmas, theorems and corollaries remain the same as in the previous section. Theorem 1 is derived from Axioms 1 and 2. Corollary 1 is derived from Axiom 3 and Theorem 1. Finally, Theorem 3 can be derived from Corollary 1 and Lemma 1, which, as shown above, depends only on Axiom 3 and Definition 1. Therefore, confirming Hájek's claim, Axioms 4 and 5 and Definitions 2 and 3 are superfluous: they are not needed to prove Theorem 3.

\subsection{A Proof-Theoretical Explanation for the Immunity to Modal Collapse}

Anderson's change in the definition of essence adds an extra box modality, which transforms the standard proof (shown in Section 6) of modal collapse (i.e. $\vdash A \rightarrow \square A$ ) into a proof of a tautology $(\vdash \square A \rightarrow \square A)$, as shown below.

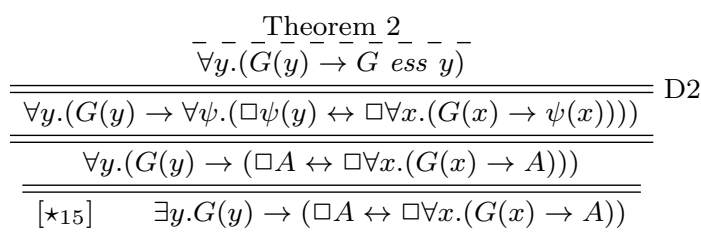




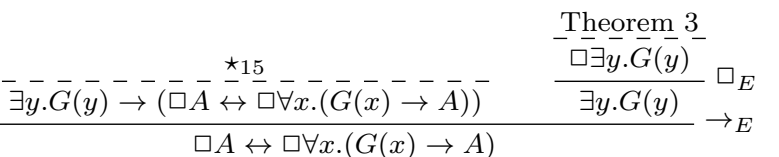

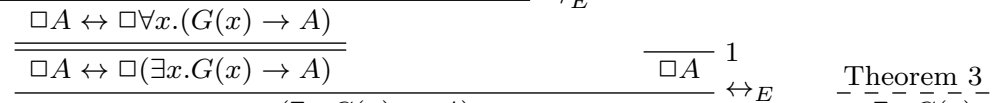

$$
\begin{aligned}
& \begin{array}{cc}
\frac{\square(\exists x \cdot G(x) \rightarrow A)}{\omega: \exists x \cdot G(x) \rightarrow A} \square_{E} & \frac{-\square \exists \bar{y} \cdot \bar{G}(\bar{y})^{-}}{\omega: \exists x \cdot G(x)} \bullet_{E} \\
\qquad \frac{\frac{\omega: A}{\square A} \square_{I}}{\square A \rightarrow \square A} \rightarrow_{I}^{1} &
\end{array}
\end{aligned}
$$

The exchange of the implication in Scott's definition of essence for an equivalence and the additional necessitation (box) modality before the property $\psi$ (which is instantiated by $\lambda x . A)$ prevents the implication of a necessary fact from its contingent counterpart, as seen in the second part of the proof above, because the hypothetical assumption of the contingent property $A$ has to be exchanged for its necessitation $\square A$ to preserve the proof structure.

\section{Bjørdal's Variant in Natural Deduction}

Bjørdal's variant (Bjørdal, 1999; Fuhrmann, 2005) is another solution to the Modal Collapse. In striking contrast to other variants, Bjørdal's axiomatization takes god-likeness $\left(G_{B}\right)$ as a primitive notion, upon which the notion of positive property is defined. This variant also significantly reduces the number of necessary axioms. Scott's variants of Axioms 2, 3 and 4 become unnecessary to prove the final theorem from Bjørdal's modified Definitions 1, 2 and 3 and Axioms 1 and 5.

Definition 1 (Changed) A property is positive iff it is necessarily possessed by every God-like being.

$$
P_{B}(\varphi) \equiv \square \forall x \cdot\left(G_{B}(x) \rightarrow \varphi(x)\right)
$$

Bjørdal refines the notion of essence by restricting the attention to positive properties only. He calls this refined notion of essence a maximal composite.

Definition 2 (Changed) A maximal composite of an individual's positive properties is a positive property possessed by the individual and necessarily implying every positive property possessed by the individual.

$$
\operatorname{MCP}(\varphi, x) \equiv\left(\varphi(x) \wedge P_{B}(\varphi)\right) \wedge \forall \psi \cdot\left(\left(\psi(x) \wedge P_{B}(\psi)\right) \rightarrow \square \forall y \cdot(\varphi(y) \rightarrow \psi(y))\right)
$$

Definition 3 (Changed) Necessary existence of an individual is the necessary exemplification of all its maximal composites.

$$
N E_{B}(x) \equiv \forall \varphi \cdot(M C P(\varphi, x) \rightarrow \square \exists y \cdot \varphi(y))
$$

Axiom 1 (Changed) If a property is positive, its negation is not positive:

$$
\forall \varphi .\left(P_{B}(\varphi) \rightarrow \neg P_{B}(\neg \varphi)\right)
$$

Axiom 5 Necessary existence is a positive property.

$$
P_{B}\left(N E_{B}\right)
$$

The proof of the possible instantiation of $G_{B}$ is adjusted (as shown below) by proving Theorem 1 of section 5 directly for $G_{B}$ and not for positive properties in general.

Theorem 1 If God-likeness is a positive property, then it is possibly exemplified:

$$
P\left(G_{B}\right) \rightarrow \diamond \exists x \cdot G_{B}(x)
$$




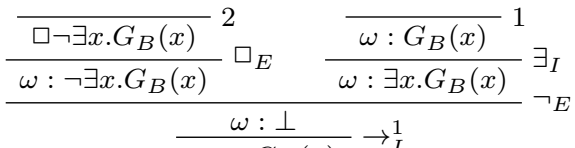

$$
\begin{aligned}
& \frac{\omega: \perp}{\omega: \neg G_{B}(x)} \rightarrow \frac{1}{I} \\
& \frac{\frac{\omega: G_{B}(x) \rightarrow \neg G_{B}(x)}{\omega: \forall x .\left(G_{B}(x) \rightarrow \neg G_{B}(x)\right)} \rightarrow_{I}}{\square_{I}} \\
& \square \forall x .\left(G_{B}(x) \rightarrow \neg G_{B}(x)\right) \\
& \stackrel{P\left(\neg G_{B}\right)}{\neg P\left(G_{B}\right)} \\
& -{ }_{-} \text {Axiom } 1 \\
& \frac{\forall \varphi \cdot(P(\neg \bar{\varphi}) \rightarrow \neg \bar{P}(\bar{\varphi}))}{P\left(\neg G_{B}(x)\right) \rightarrow \neg P\left(G_{B}\right)} \forall_{E}
\end{aligned}
$$

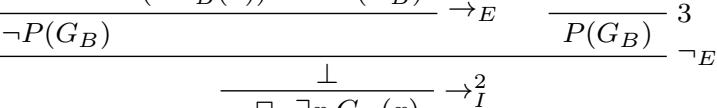

$$
\begin{aligned}
& \frac{\perp}{\neg \square \neg \exists x \cdot G_{B}(x)} \rightarrow_{I}^{2} \\
& \frac{\overline{\diamond \exists x \cdot G_{B}(x)}}{P\left(G_{B}\right) \rightarrow \diamond \exists x \cdot G_{B}(x)} \rightarrow I_{I}^{3}
\end{aligned}
$$

Corollary 1 Possibly, a God-like being exists:

$$
\diamond \exists x \cdot G_{B}(x)
$$

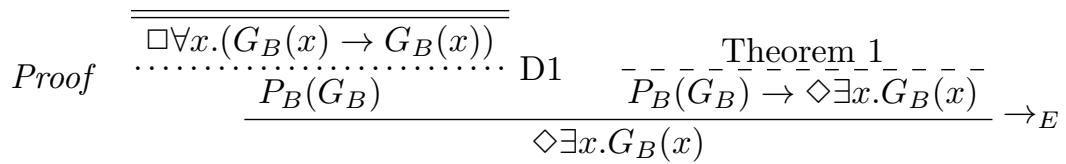

Lemma 1 If there is a God-like being, then there is a God-like being necessarily:

$$
\exists z \cdot G_{B}(z) \rightarrow \square \exists x \cdot G_{B}(x)
$$

Proof

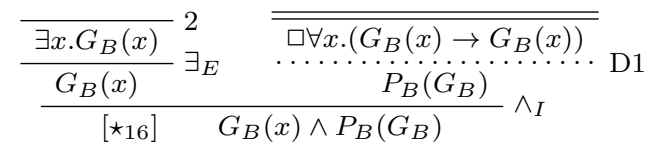

$$
\begin{aligned}
& \overline{\frac{\left(\psi(x) \wedge P_{B}(\psi)\right)}{P_{B}(\psi)}} \wedge_{E} \\
& \frac{\frac{\square \forall y \cdot\left(G_{B}(y) \rightarrow \psi(y)\right)}{\left(\psi(x) \wedge P_{B}(\psi)\right) \rightarrow \square \forall y \cdot\left(G_{B}(y) \rightarrow \psi(y)\right)}}{-G_{B}(x) \wedge P_{B}^{\star}\left(G_{B}\right)} \rightarrow_{I}^{1} \\
& \text { Axiom } 5 \\
& \bar{P}_{B}\left(\bar{N} \bar{E}_{B}\right) \\
& \square \forall x .\left(G_{B}(x) \rightarrow N E_{B}(x)\right) \text { D1 }
\end{aligned}
$$

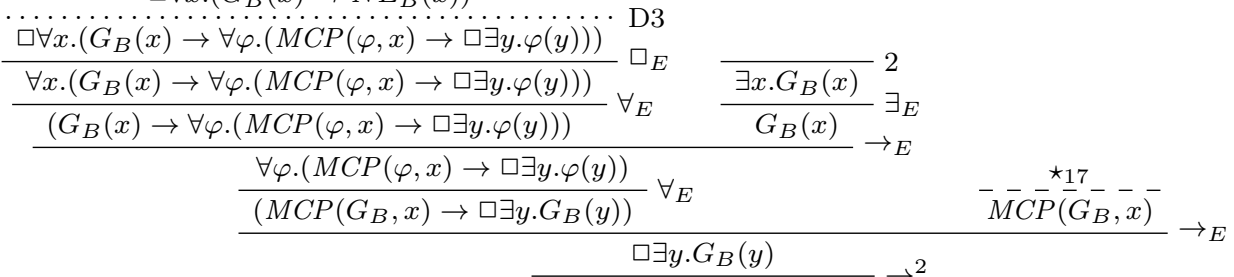

$$
\begin{aligned}
& \frac{\square \exists y \cdot G_{B}(y)}{\exists x \cdot G_{B}(x) \rightarrow \square \exists y \cdot G_{B}(y)} \rightarrow_{I}^{2}
\end{aligned}
$$




\section{Conclusions}

The formal proofs presented here are short and accessible because of the choice of a natural deduction calculus. The new proof of section 5 shows explicitly that the weaker modal logic KB is sufficient for the ontological argument. The stronger modal logic S5, which is usually taken for granted in discussions of Gödel's ontological argument, is not necessary. This weakening of the underlying logic confirms and strengthens a claim briefly mentioned by Anderson (Anderson, 1990, footnote 2). KB is also sufficient for the variants of Anderson and Bjørdal, which address Sobel's criticism of the modal collapse.

Acknowledgements: We thank Padre Edvaldo (from Piracicaba, Brazil) for assisting Bruno and his family in a moment of need. Gratitude for his assistance is what triggered the production of an initial draft of this article in early 2013. We are thankful to Christoph Benzmüller for collaborations on several related articles. This work was supported by a Stipendium of the Österreichische Akademie der Wissenschaften (APART).

\section{References}

Adams, R. M. 1995. Introductory note to [Gödel 1970].

Anderson, C. A. 1990. Some Emendations of Gödel's Ontological Proof. Faith and Philosophy, Vol. 7, Issue 3, pp. 291-303.

Anderson, C. A.\& Gettings, M. 1996. Gödel's ontological proof revisited. In: edited by Hájek P. Gödel '96, Springer.

Bentert, M. \& Benzmüller, C. \& Streit, D. \& Woltzenlogel Paleo, B. 2016. Analysis of an Ontological Proof Proposed by Leibniz. [to appear in] Death and Anti-Death, Volume 14: Four Decades after Michael Polanyi, Three Centuries after G. W. Leibniz. Ed. Tandy, C. Ria University Press.

Benzmüller, C. \& Brown C. \& Kohlhase, M. In: Journal of Symbolic Logic, Vol. 69, N. 4, pp. 1027-1088.

Benzmüller, C. \& Weber, L. \& Woltzenlogel Paleo, B. 2015. Computer-Assisted Analysis of the Anderson-Hájek Ontological Controversy. Handbook of the 1st World Congress on Logic and Religion. Eds. Silvestre, R. S. \& Béziau, J-Y.

Benzmüller, C. \& Weber, L. \& Woltzenlogel Paleo, B. 2017. Computer-Assisted Analysis of the Anderson-Hájek Ontological Controversy. To appear in: Logica Universalis Volume 11, Issue 1. Eds. Silvestre, R. S. \& Béziau, J-Y. Springer. pp. 1-11.

Benzmüller, C. \& Woltzenlogel Paleo, B. 2013a. Gödel's God on the Computer. Proceedings of the 10th International Workshop on the Implementation of Logics. Eds. Schulz, S.; Sutcliffe, G. and Konev, B. pp. 1-2.

Benzmüller, C. \& Woltzenlogel Paleo, B. 2013b. Gödel's God in Isabelle/HOL. Archive of Formal Proofs.

Benzmüller, C. \& Woltzenlogel Paleo, B. 2014a. On Logic Embeddings and Gödel's God. Procedings WADT 2014: Revised Selected Papers of the 22nd International Workshop on Algebraic Development Techniques. Eds. Codescu, M. et al. Recent Trends in Algebraic Development Techniques. Springer-Verlag New York. pp. 3-6.

Benzmüller, C. \& Woltzenlogel Paleo, B. 2014b. On Logic Embeddings and Gödel's God. Preliminary Proceedings of the 22nd International Workshop on Algebraic Development Techniques. Eds. Diaconescu, R. et al. pp. 8-9.

Benzmüller, C. \& Woltzenlogel Paleo, B. 2014c. Formalization and Automated Verification of Gödel's Proof of God's Existence. Handbook of the World Congress on the Square of Opposition IV. Eds. Béziau, J-Y. \& Gan-Krzywoszyrinska, K. pp. 24-25.

Benzmüller, C. \& Woltzenlogel Paleo, B. 2014d. Automating Gödel's Ontological Proof of God's Existence with Higher-order Automated Theorem Provers. Proceedings ECAI 2014 
- 21st European Conference on Artificial Intelligence- Including Prestigious Applications of Intelligent Systems (PAIS 2014). Eds. Schaub, T. et al. pp. 93-98.

Benzmüller, C. \& Woltzenlogel Paleo, B. 2015a. Interacting with Modal Logics in the Coq Proof Assistant. Computer Science - Theory and Applications - Proceedings of the 10th International Computer Science Symposium in Russia, CSR 2015. Lecture Notes in Computer Science. Eds. Beklemishev, L. D. \& Musatov, D. V. Springer. pp. 398-411.

Benzmüller, C. \& Woltzenlogel Paleo, B. 2015b. Higher-Order Modal Logics: Automation and Applications. Reasoning Web. Web Logic Rules - 11th International Summer School 2015. Tutorial Lectures. Lecture Notes in Computer Science. Eds. Faber, W. and Paschke, A. Springer. pp. $32-74$.

Benzmüller, C. \& Woltzenlogel Paleo, B. 2015c. Experiments in Computational Metaphysics: Gödel's Proof of God's Existence. Science and Spiritual Quest: Proceedings of the 9th All India Students' Conference on Science and Spiritual Quest. Vol. 9. Eds. Mishra, S.C.; Ghosh, S. and Agarwal, V. Bhaktivedanta Institute. pp. 23-40.

Benzmüller, C. \& Woltzenlogel Paleo, B. 2016a. The Inconsistency in Gödel's Ontological Argument: A Success Story for AI in Metaphysics. Proceedings of the Twenty-Fifth International Joint Conference on Artificial Intelligence - IJCAI. Ed. Kambhampati, S. AAAI Press.

Benzmüller, C. \& Woltzenlogel Paleo, B. 2016b. The Modal Collapse as a Collapse of the Modal Square of Opposition. Studies in Universal Logic. The Square of Opposition: A Cornerstone of Thought. Eds. Béziau, J-Y. and Basti, G. Springer. pp. 1-7.

Bjørdal, F. 1999. Understanding Gödel's Ontological Argument. Ed. Childers, T. The Logica Yearbook 1998, pp. 214-217, Filosofia.

Blackburn, P. \& M. de Rijke \& Y. Venema. 2001. Modal Logic. Cambridge: Cambridge University Press.

Church, A. 1940. A Formulation of the Simple Theory of Types, Journal of Symbolic Logic, 5: $56-68$.

Fitting, M. \& Mendelsohn, R. 1998. First-order Modal Logic, Synthese Library vol. 277. Kluwer Academic Publishers.

Fitting, M. 2002. Types, Tableaus, and Gödel's God, Kluwer Academic Publishers.

Fuhrmann, A. 2005. Existenz und Notwendigkeit - Kurt Gödel's Axiomatische Theologie. Eds. Olsson e.J., Schröder-Heister, P. and Spohn, W. Logik in der Philosophie, pp. 349374, Synchr.-Wissenschafts-Verlag.

Gabbay, D. 1996. Labelled Deductive Systems: volume I. New York: Oxford Science publications.

Gödel, K. Ontological Proof. In Kurt Gödel Collected Works vol. III. Ed. Feferman, S. et al., pp. 403-404, 139, 145 or Appendix A. Notes in Kurt Gödel's hand in [Sobel 2001].

Hájek, P. 1996. Magari and others on Gödel's Ontological Proof. Eds. Ursini et al. Logic and Logical Algebra, pp. 125-136, Marcel Dekker.

Kant, I. original 1781. Critique of Pure Reason, J. M. Dent \& Sons LTD, edition from 1959.

Koons, R. 2006. Sobel on Gödel's Ontological Proof. Philosophia Christi, 2:235-248.

Magari, R. 1988. Logica e Teofilia. In: Notizie di Logica VII.4.

Muskens, R. 2006. Higher Order Modal Logic. Eds. Blackburn, P. \& van Benthem, J.F.A.K. \& Wolter, F. Handbook of Modal Logic, Studies in Logic and Practical Reasoning, pages 621-653. Elsevier, Dordrecht, 2006.

Nipkow, T. \& Paulson, L.C. \& Wenzel, M. 2002. Isabelle/HOL: A Proof Assistant for HigherOrder Logic. Number 2283 in LNCS, Springer.

Oppenheimer, P. \& Zalta, E. 2011. A Computationally-Discovered Simplification of the Ontological Argument. Australasian Journal of Philosophy, 89(2), 333-349.

Paulin-Mohring, C. 2015. Introduction to the calculus of inductive constructions. Eds. Delahaye, D. \& Woltzenlogel Paleo, B. All about Proofs, Proofs for All, Mathematical Logic and Foundations. College Publications, London. 
Prawitz, D. 2006 (1st ed. 1965). Natural deduction: a proof-theoretical study. Mineola, New York: Dover publications.

Rushby, J. 2013. The Ontological Argument in PVS. Proceedings of CAV Workshop "Fun with Formal Methods". St. Petersburg. Ed. Shilov, N.

Scott, D. Appendix B. Notes in Dana Scott's hand in Logic and Theism: Arguments for and against Beliefs in God by Sobel, J. H.

Sobel, J. H. 1987. Gödel's Ontological Proof. In: J. J. Thompson (ed.). On being and saying: essays for Richard Cartwright, MIT Press.

Sobel, J. H. 2001. Logic and Theism: Arguments for and against Beliefs in God, Cambridge University Press.

Thiele, R. 2003. Hilbert's twenty-fourth problem, American Mathematical Monthly, January 2003.

Wang, H. 1996. A Logical Journey: From Gödel to Philosophy, The MIT Press.

Woltzenlogel Paleo, B. 2013. Automated Verification and Reconstruction of Gödel's Proof of God's Existence. Journal of the Austrian Computer Society. pp. 4-6. 